\title{
RANGE OF SAFE ZONE FROM OBSTACLES FOR UNMANNED AERIAL VEHICLE
}

\author{
L. Nocoń* ${ }^{*}$ K. Stefański***
}

\begin{abstract}
The article presents the problem of determining the safe distance of an unmanned aerial vehicle $(U A V)$ from an obstacle. Describing a safe zone will allow an effective UAV response when an obstacle occurs on the trajectory of the flight. The obstacle will be avoided if it breaches the safe zone. In operation, the safe zone is defined by means of a rotating paraboloid. Two models are presented: a safe zone in the horizontal plane and in 3D space. These methods can be successfully used for unmanned ground vehicles.
\end{abstract}

Keywords: unmanned aerial vehicle (UAV), safe zone, avoiding obstacles

\section{Introduction}

UAVs are used for military purposes, as well as for reconnaissance and observation of urban areas. They are increasingly used in commercial and recreational applications. The ability of the UAV to avoid obstacles will minimize the possibility of damage during performing the mission objectives. It will also significantly affect the safety of users and third parties.

Detecting an obstacle and avoiding it without unnecessary stopping will allow for performing the chosen trajectory quickly and efficiently. The most common task of the UAV is to make a flight on a specific trajectory. The goals of such a flight may include a fire mission, reconnaissance or tracking, and a target for other operations. During the flight along the trajectory, the UAV may encounter unexpected obstacles not included in the program trajectory. The device must then avoid the obstacle and return to the original trajectory (Helgason et al., 2001). Determination of a safe zone (Fig.1) will allow the UAV to avoid an obstacle in good time.

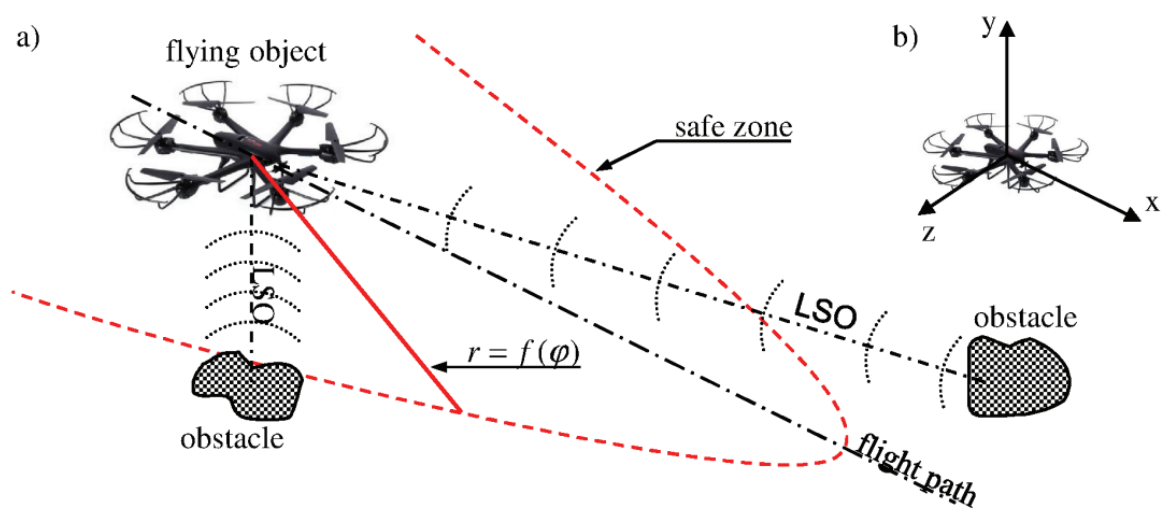

Fig. 1: Overview of the location of the safe zone, location of the line of sight; b) axis orientation of the coordinate systems related to the object

Research Assistant Łukasz Nocoń, Ph.D. Eng.: Faculty of Mechatronics and Mechanical Engineering, Kielce University of Technology, al. Tysiąclecia P.P. 7; 25-314, Kielce; PL, lnocon@tu.kielce.pl

** Assistant Prof. Konrad Stefański, Ph.D. Eng.: Faculty of Mechatronics and Mechanical Engineering, Kielce University of Technology, al. Tysiąclecia P.P. 7; 25-314, Kielce; PL, kstefanski@tu.kielce.pl 
For multirotors, the frequent method of avoiding obstacles is the use of composite bumpers made of carbon fiber or other fabrics (Barchan, 2008 and Chatys et al., 2017). It is a contact method and prevents the multirotor's destruction in case of direct contact with an obstacle. This solution is unacceptable for UAV airframes. The proposed methods are non-contact methods. Avoiding obstacles by detecting distances will minimize the possibility of UAV damage. The obstacle detection can be carried out with the help of a modified tracking head (Gapiński et al., 2014).

\section{Safe zone determination}

A safe zone can be set in many ways. Determination of the appearance of the zone depends on the requirements of the UAV. The zone can take the shape of an ellipse, a parabola, a rectangle, a circle, etc. A zone drawn out with a parabola is proposed in the paper. Two cases are considered. In the first case, we deal with the zone in the horizontal plane. In the second case, a method for determining a safe zone in 3D space is developed. The safe zone is formed by the surface of a rotating paraboloid.

The vertex of the parabola $q$ (frontal distance), which is the distance from the obstacle on the flight path, varies depending on the velocity of the UAV. A multicopter whose flight velocity is relatively small needs a smaller distance to perform the maneuver avoiding the obstacle. A missile, which moves at a velocity of approx. $250 \mathrm{~m} / \mathrm{s}$ and its inertia and limited maneuverability makes it difficult to omit an obstacle, needs a relatively greater distance to react. It is assumed that the side safe zone for UAV is $1 \mathrm{~m}$.

\subsection{Obstacles in the horizontal plane}

In many cases it is sufficient to detect obstacles only in the horizontal plane. For slow moving UAVs (multirotors in open spaces) it is not necessary to scan the vertical plane. However, this method is mainly useful for ground objects.

The safe zone is described by a parabola (1) in the range $z \in\langle-1 ; 1\rangle$ :

$$
x=f(z)=-q z^{2}+q
$$

The polynomial curve $f(z)$ poles are parametrized so that the argument of the sought function $f(\varphi)$ is the angle of the $r$ vector deviation from the light path $\varphi$. The function value is the UAV distance to the curve $f(z)$ (Fig. 2).

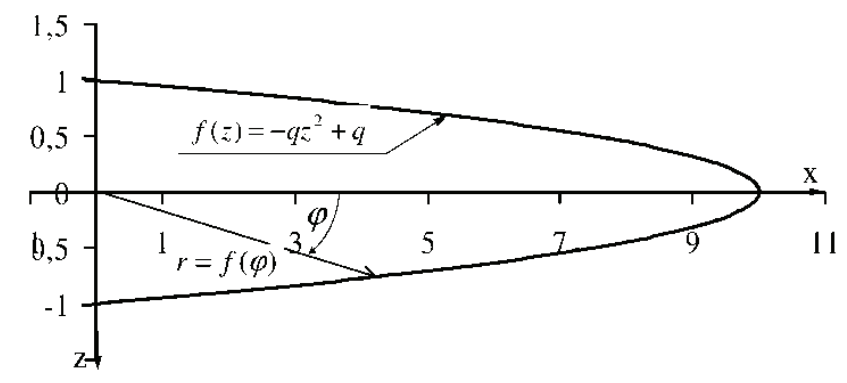

Fig. 2: Scheme of the function determining- $r=f(\varphi)$ and angle orientation- $\varphi$ for $q=10 \mathrm{~m}$.

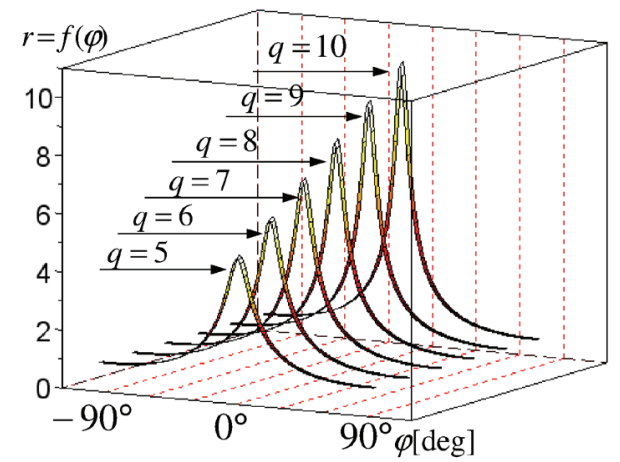

Fig. 3: Radius- $r=f(\varphi)$ in the function of azimuth angle- $\varphi$ for the frontal distance- $q=5 . .10 \mathrm{~m}$.

Based on angular dependencies (Fig. 2):

$$
x=r \cos \varphi, z=-r \sin \varphi
$$

Substituting (1) in the formula (2),we have the equation:

$$
r \cos \varphi=-q r^{2} \sin ^{2} \varphi+q
$$


Finally, the radius in the function of angles $r=f(\varphi)$ is (4) and takes values like in Fig. 3.

$$
f(\varphi)=\left\{\begin{array}{ccc}
\frac{\sqrt{q+\frac{\cos ^{2}(\varphi)}{4 q \sin ^{2}(\varphi)}-\frac{\cos (\varphi)}{2 \sqrt{q \sin ^{2}(\varphi)}}}}{\sqrt{q \sin ^{2}(\varphi)}} & \text { dla } & \varphi \neq 0 \\
q & \text { dla } & \varphi=0
\end{array}\right.
$$

\subsection{Obstacle detection in 3D space}

In 3D space, the safe distance zone depends on two angles. Two cases are considered depending on the used technology in the space search system. In the first case the key angle is the angle of deviation of the vector $r=f(\varphi)$ from the flight path $\varphi$, and the angle of $r$ vector rotation around the $O x$ axis indicates on which side of the UAV the obstacle lies (Fig. 4a). In the second case, the angles are: the deviation in the vertical plane- $y O x: \delta_{y}$, and the deviation in the horizontal plane $z O x: \delta_{z}$ (Fig. $4 \mathrm{~b}$ ). The second case is considered in the paper (Fig. 4b). In both cases, the same formula is used for the rotating paraboloid which determines the zone of safe distance.

$$
f(y, z)=-q y^{2}-q z^{2}+q
$$
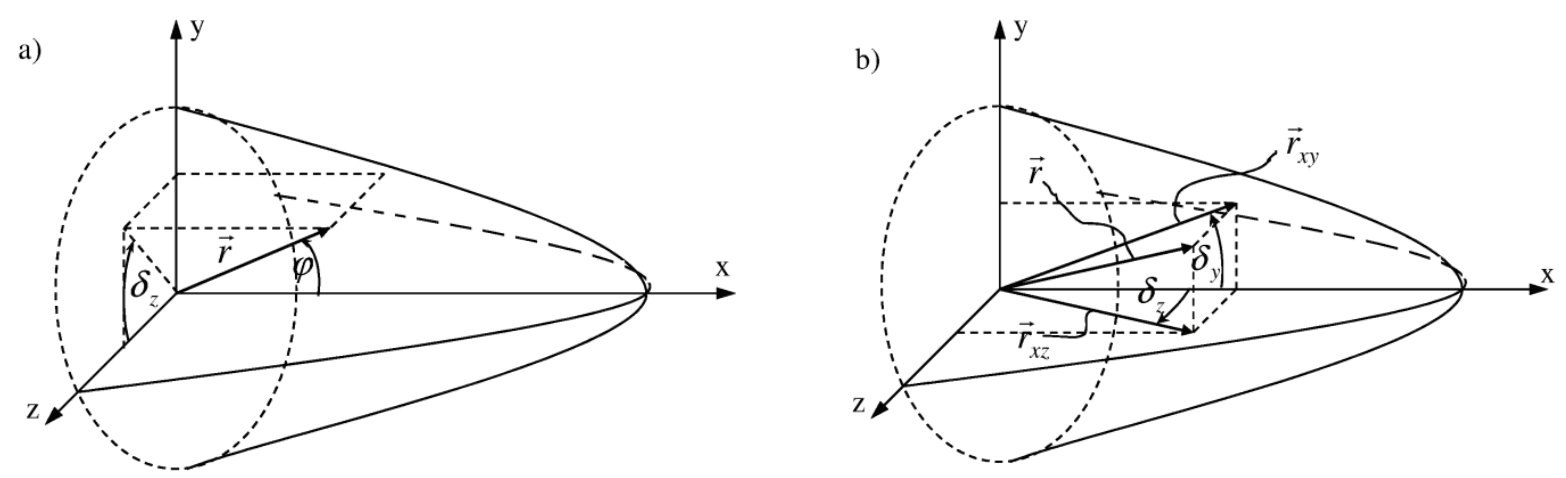

Fig. 4: Scheme of determining the zone of safe distance from an obstacle; a) for function: $r=f(\varphi)$ rotating around Ox axis; b) for function: $r=f\left(\delta_{y}, \delta_{z}\right)$;

We parametrize the variables:

$$
x=r_{x z} \cos \delta_{z} ; x=r_{x y} \cos \delta_{y} ; y=r_{x y} \sin \delta_{y} ; z=r_{x z} \sin \delta_{z}
$$

The purpose is to have the function of the radius length- $r=f\left(\delta_{y}, \delta_{z}\right)$. Based on the formula for the radius length: $r=\sqrt{x^{2}+y^{2}+z^{2}}$ we have two equations with two unknowns

$$
\left\{\begin{array}{l}
r^{2}=r_{x z}^{2} \sin ^{2} \delta_{z}+r_{x y}^{2} \\
r^{2}=r_{x y}^{2} \sin ^{2} \delta_{y}+r_{x z}^{2}
\end{array}\right.
$$

Whose solution is a pair of equations:

$$
\left\{\begin{array}{l}
r_{x z}^{2}=\frac{r^{2} \cos ^{2} \delta_{y}}{1-\sin ^{2} \delta_{y} \sin ^{2} \delta_{z}} \\
r_{x y}^{2}=\frac{r^{2} \cos ^{2} \delta_{z}}{1-\sin ^{2} \delta_{y} \sin ^{2} \delta_{z}}
\end{array}\right.
$$

After substituting in the formula (5), there is 


$$
q \frac{r^{2} \cos ^{2} \delta_{z} \sin ^{2} \delta_{y}}{1-\sin ^{2} \delta_{y} \sin ^{2} \delta_{z}}+q \frac{r^{2} \cos ^{2} \delta_{y} \sin ^{2} \delta_{z}}{1-\sin ^{2} \delta_{y} \sin ^{2} \delta_{z}}=5-\frac{r \cos \delta_{y} \cos \delta_{z}}{\sqrt{1-\sin ^{2} \delta_{y} \sin ^{2} \delta_{z}}}
$$

Finally, the radius in the function of angles- $r=f\left(\delta_{y}, \delta_{z}\right)$ is (10) and takes values like in fig. 5,6.:

$$
f\left(\delta_{y}, \delta_{z}\right)=\left\{\begin{array}{cc}
\frac{\sqrt{q+\frac{\cos ^{2} \delta_{z} \cos ^{2} \delta_{y}}{4 q\left(\sin ^{2} \delta_{y} \cos ^{2} \delta_{z}+\sin ^{2} \delta_{z} \cos ^{2} \delta_{y}\right)}-\frac{\cos \delta_{z} \cos \delta_{y}}{2 \sqrt{q\left(\sin ^{2} \delta_{y} \cos ^{2} \delta_{z}+\sin ^{2} \delta_{z} \cos ^{2} \delta_{y}\right)}}}}{\sqrt{\frac{q\left(\sin ^{2} \delta_{y} \cos ^{2} \delta_{z}+\sin ^{2} \delta_{z} \cos ^{2} \delta_{y}\right)}{1-\sin ^{2} \delta_{y} \sin ^{2} \delta_{z}}}} & \delta_{y}, \delta_{z} \neq 0 \\
q & \delta_{y}, \delta_{z}=0
\end{array}\right.
$$

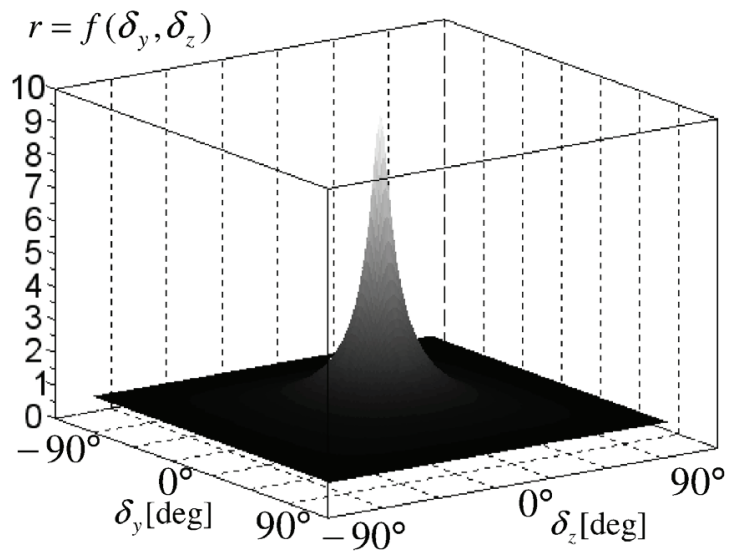

Fig. 5: Radius- $r=f\left(\delta_{y}, \delta_{z}\right)$ in the function of azimuth angle- $\delta_{z}$ and elevation angle- $\delta_{y}$ for the frontal distance- $q=10 \mathrm{~m}$.

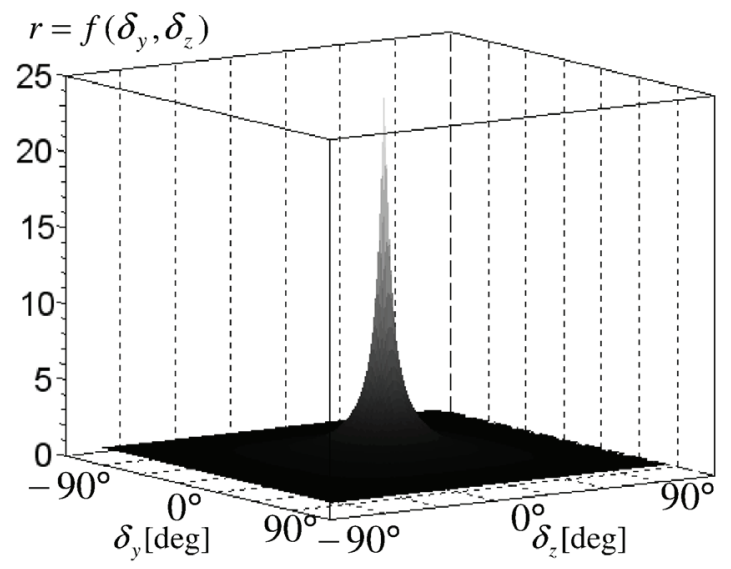

Fig. 6: Radius- $r=f\left(\delta_{y}, \delta_{z}\right)$ in the function of azimuth angle $\delta_{z}$ and elevation angle $\delta_{y}$ for the frontal distance $q=25 \mathrm{~m}$

\section{Conclusions}

The presented solutions relate to relatively small UAVs with a span of no more than $1 \mathrm{~m}$. These are preliminary studies that show a general approach to the problem of obstacles detection and avoidance. The method can be applied to larger UAVs by changing parabola parameters.

Information for the autopilot, when the obstacle becomes a threat to the UAV, is determined by the distance of the safe zone border. The space search system scans the nearby surroundings to detect a potential obstacle. A detected object that does not violate the safe zone is not a threat. To this end, a $r$ radius is defined to determine for which angle position in relation to the UAV the object becomes an obstacle. The measured distance from a potential obstacle is compared with the $r$ safe distance for the obstacle's angles of view. If the distance is greater than $r$, the potential obstacle does not violate the zone.

\section{References}

Barchan, A. and Chatys, R.(2008) Experimental Verification of Some Elastic Properties of Unidirectional Composites. Mechanics of Composite Materials, vol. 44, No. 2, 2008 - pp. 131-138.

Chatys, R., Miśków, G. and Miśków, J. (2017) Structural modelling of the strength properties of polymer composites, in: Engineering Mechanics 2017, Brno University of Technology, Brno, pp. 250-254.

Gapiński, D., Krzysztofik, I. and Koruba, Z. (2014) Analysis of the dynamics and control of the modified optical target seeker used in anti-aircraft rocket missiles. Journal of Theoretical and Applied Mechanics, 52, 3, pp. 629639

Helgason, R.V., Kennington, J.L. and Lewis, K.R. (2001) Cruise Mission Planning: A Heuristic Algorithm for Automatic Path Generation. Journal of Heuristics, 7, pp. 473-494. 